\title{
TRIPOSES AS A GENERALIZATION OF LOCALIC GEOMETRIC MORPHISMS
}

\author{
JONAS FREY AND THOMAS STREICHER
}

\begin{abstract}
In HJP80 Hyland, Johnstone and Pitts introduced the notion of tripos for the purpose of organizing the construction of realizability toposes in a way that generalizes the construction of localic toposes from complete Heyting algebras. In Pit02 one finds a generalization of this notion eliminating an unnecessary assumption of [HJP80].

The aim of this paper is to characterize triposes over a base topos $\mathcal{S}$ in terms of so-called constant objects functors from $\mathcal{S}$ to some elementary topos. Our characterization is slightly different from the one in Pitts's PhD Thesis Pit81 and motivated by the fibered view of geometric morphisms as described in [Str20]. In particular, we discuss the question whether triposes over Set giving rise to equivalent toposes are already equivalent as triposes.
\end{abstract}

Dedicated to the Memory of Martin Hofmann

\section{INTRODUCTION}

As described in [Joh77] localic geometric morphisms to a topos $\mathcal{S}$ are given by functors $F$ from $\mathcal{S}$ to some topos $\mathcal{E}$ such that

(1) $F$ preserves finite limits,

(2) every object $A \in \mathcal{E}$ appears as subquotient of some $F I$ and

(3) $F$ has a right adjoint.

In the appendix of [Str20] one finds a proof of M. Jibladze's Theorem [Jib88] saying that fibered toposes over $\mathcal{S}$ having internal sums correspond to finite limit preserving functors from $\mathcal{S}$ to some topos $\mathcal{E}$. In particular, a finite limit preserving functor $F: \mathcal{S} \rightarrow \mathcal{E}$ gives rise to the fibration $P_{F}=F^{*} P_{\mathcal{E}}$ over $\mathcal{S}$ obtained by change of base along $F$ from the fundamental ("codomain") fibration $P_{\mathcal{E}}=\operatorname{cod}: \mathcal{E}^{\mathcal{D}} \rightarrow \mathcal{E}$ (where $\mathcal{Z}$ is the small category $0 \rightarrow 1$ corresponding to the ordinal 2). But every fibered topos $P: \mathcal{X} \rightarrow$ Set with internal sums is equivalent to $P_{\Delta}$ where $\mathcal{E}$ is the fiber of $P$ over 1 and $\Delta: \mathcal{S} \rightarrow \mathcal{E}$ sends $I \in \mathcal{S}$ to $\Delta(I)=\coprod_{I} 1_{I}$ in $\mathcal{E}$.

Moreover, as also shown in Str20] for terminal object preserving $F: \mathcal{S} \rightarrow \mathcal{E}$ the fibration $P_{F}$ is locally small iff $F$ has a right adjoint. Thus, as observed by J. Bénabou already in his 1974 Montreal lectures [Bén74], inverse image parts of geometric morphisms correspond to terminal object preserving functors $F$ between toposes such that the fibration $P_{F}$ has internal sums and is locally small.

Moreover, as also observed in $\underline{\text { Str20 }}$ for a finite limit preserving functor $F: \mathcal{S} \rightarrow$ $\mathcal{E}$ between toposes condition (2) is equivalent to the requirement that every map 
$a: A \rightarrow F I$ in $\mathcal{E}$ fits into a commuting diagram

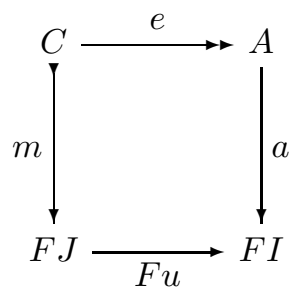

where $e$ is epic and $m$ is monic. Obviously, this condition entails (2) instantiating $I$ by a terminal object. For the reverse direction choose $m: C \longmapsto F J$ and $e: C \rightarrow A$ (which exist by condition (2)) and observe that

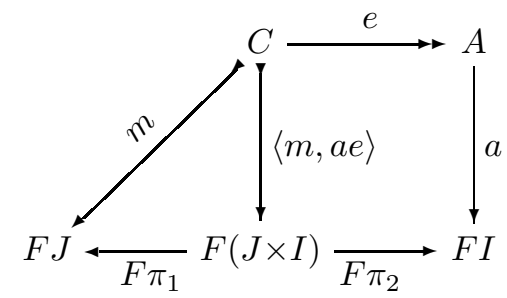

using the assumption that $F$ preserves finite limits and thus finite products. Thus, condition (2) amounts to the requirement that every object of $\mathcal{E}$ can be covered by a(n internal) sum of subterminals (in the appropriate fibrational sense!). As observed in Str20] under assumption (3) this is equivalent to the requirement that $g$ in

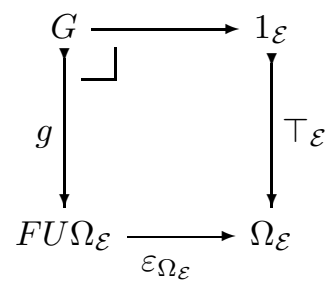

is a generating family for the fibration $P_{F}$ (where $U$ is right adjoint to $F$ ).

\section{A fibrational aCCOUnt of triposes}

In HJP80 Hyland, Johnstone and Pitts have identified a notion of fibered preorder $\mathscr{P}$ over a base topos $\mathcal{S}$ giving rise to a topos $\mathcal{S}[\mathscr{P}]$ by "adding subquotients" related to the base topos via a constant object functor $\Delta_{\mathscr{P}}: \mathcal{S} \rightarrow \mathcal{S}[\mathscr{P}]$ satisfying conditions (1) and (2) of the previous section. As obvious from the considerations in loc.cit. one may get back the fibered preorder $\mathscr{P}$ from the subobject fibration Sub $_{\mathcal{S}[\mathscr{P}]}$ by change of base along $\Delta_{\mathscr{P}}$. For this reason such $\mathscr{P}$ were called "topos representing indexed pre-ordered sets" suggesting the acronym "tripos" (echoing the traditional name for final exams at University of Cambridge).

The original definition in HJP80 required triposes to have a generic family, i.e. a $T \in \mathscr{P}(\Sigma)$ from which all objects in $\mathscr{P}(I)$ may be obtained (up to isomorphism) by reindexing along an appropriate (generally not unique) map $I \rightarrow \Sigma$. In [Pit02], Pitts observed that the requirement of a generic family can be replaced by a weaker 'comprehension axiom' [Pit02, Axiom 4.1] which still implies - and is actually 
equivalent to - the fact that the category $\mathcal{S}[\mathscr{P}]$ of partial equivalence relations is a topos 1 .

In this work we focus on triposes in this more general sense, and contrary to the literature we use the word 'tripos' not for the fibered preorder, but for the associated constant objects functor (from which the fibered preorder can be reconstructed as pointed out above). This way we obtain a definition that is a straightforward generalization of the notion of localic geometric morphism as presented in the introduction. Pitts's comprehension axiom is then presented as Lemma 2.2. We refer to (constant objects functors arising from) the more restrictive notion of [HJP80] as "traditional triposes":

Definition 2.1. A tripos over a topos $\mathcal{S}$ is a finite limit preserving functor $F$ from $\mathcal{S}$ to a topos $\mathcal{E}$ such that every $A \in \mathcal{E}$ appears as subquotient of $F I$ for some $I \in \mathcal{S}$.

$A$ tripos is called traditional if the fibered preorder $\mathscr{P}_{F}=F^{*} \operatorname{Sub}_{\mathcal{E}}$ admits a generic family, i.e. there is a a monot $: T \longmapsto F \Sigma$ such that every mono $m: P \longmapsto F I$ fits into a pullback diagram

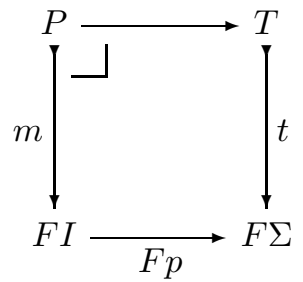

for some (typically not unique) $p: I \rightarrow \Sigma$.

The fibered preorder $\mathscr{P}_{F}$ is a first-order hyperdoctrine, i.e. a fibration of Heyting algebras with internal sums and products, since $\operatorname{Sub}_{\mathcal{E}}$ has and change of base along the finite limit preserving functor $F$ preserves the required properties (see [Str20]). Recalling Pitts's proof of the comprehension axiom, we now show that $\mathscr{P}_{F}$ admits an interpretation higher order (intuitionistic) logic.

Lemma 2.2. For every tripos $F: \mathcal{S} \rightarrow \mathcal{E}$, the fibered preorder $\mathscr{P}_{F}$ satisfies the following comprehension axiom.

$(\mathrm{CA})$ For every object $I$ in $\mathcal{S}$ there is are objects $P(I)$ in $\mathcal{S}$ and $\varepsilon_{I} \in \mathscr{P}_{F}(I \times P(I))$ such that for all $J$ in $\mathcal{S}$ and $\rho \in \mathscr{P}_{F}(I \times J)$, the formula

$$
\forall j \in J . \exists p \in P(I) . \forall i \in I . \rho(i, j) \leftrightarrow i \varepsilon_{I} p
$$

holds in the internal logic of $\mathscr{P}_{F}$.

Proof. Let $I$ be an object of $\mathcal{S}$. Then since $\mathscr{P}$ is a tripos there is an object $P(I)$ in $\mathcal{S}$ such that $\mathcal{P}(F I)$ appears as subquotient of $F(P(I))$, i.e. there is a subobject $m_{I}: C_{I} \longmapsto F(P(I))$ such that there exists an epi $e_{I}: C_{I} \rightarrow \mathcal{P}(F I)$. Consider

\footnotetext{
${ }^{1}$ The definition of tripos in Pit02 generalizes that of [HJP80] also in the sense that it admits arbitrary finite-product categories as base categories, and requires the Beck-Chevalley condition (BC) only for certain pullback squares definable from finite products. However, in the present work we consider only toposes as base categories, and all triposes satisfy BC for arbitrary pullback squares.
} 


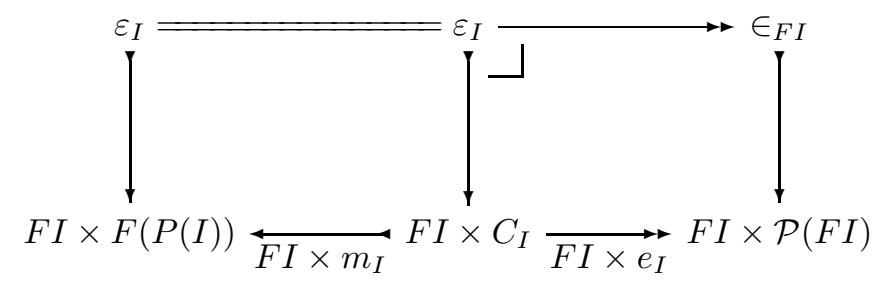

giving rise to a subobject $\varepsilon_{I}$ of $F(I \times P(I)) \cong F I \times F(P(I))$. Since the left square in the above diagram is a pullback we have $\left(F I \times m_{I}\right)^{*} \varepsilon_{I}=\left(F I \times e_{I}\right)^{*} \in_{F I}$.

Suppose $\rho: R \longmapsto F(I \times J) \cong F I \times F J$. Then

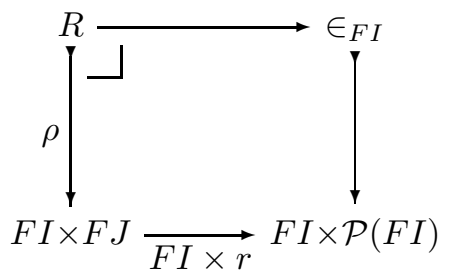

for a unique $r: F J \rightarrow \mathcal{P}(F I)$. Consider the pullback

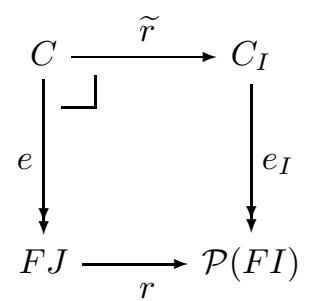

where $e$ is epic since in a topos epis are stable under arbitrary pullbacks. Thus, we have

$$
\begin{aligned}
& (F I \times e)^{*} \rho=(F I \times e)^{*}(F I \times r)^{*} \in_{F I} \cong(F I \times e r)^{*} \in_{F I}= \\
& =\left(F I \times e_{I} \widetilde{r}\right)^{*} \in_{F I} \cong(F I \times \widetilde{r})^{*}\left(F I \times e_{I}\right)^{*} \in_{F I}= \\
& \cong\left(F I \times m_{I} \widetilde{r}\right)^{*} \varepsilon_{I} \\
& =(F I \times \widetilde{r})^{*}\left(F I \times m_{I}\right)^{*} \varepsilon_{I} \cong
\end{aligned}
$$

from which it readily follows that

$$
\forall j \in J . \exists p \in P(I) . \forall i \in I . \rho(i, j) \leftrightarrow i \varepsilon_{I} p
$$

holds in the internal logic of $\mathscr{P}_{F}$.

From Lemma 2.2 and the results of $[$ Pit02] it follows that fibered preorders of the form $F^{*} \operatorname{Sub}_{\mathcal{E}}$ for some tripos $F: \mathcal{S} \rightarrow \mathcal{E}$ may up to equivalence be characterized as Heyting algebras $\mathscr{P}$ fibered over $\mathcal{S}$ with internal sums $\exists$ and internal products $\forall$ satisfying the comprehension axiom (CA).

A tripos $F: \mathcal{S} \rightarrow \mathcal{E}$ is traditional iff $\mathscr{P}_{F}$ is a tripos in the sense of [HJP80, i.e. there exists a $T \in \mathscr{P}(\Sigma)$ from which all $P \in \mathscr{P}(I)$ can be obtained by reindexing along some map $p: I \rightarrow \Sigma$.

If a tripos $F$ has a right adjoint (and thus is the inverse image part of a localic geometric morphism) then it is always traditional since we can set $\Sigma=U \Omega_{\mathcal{E}}$, and in this case the maps $p$ are unique. 
Finally, we note that $F$ is the inverse image part of a localic geometric morphism iff $F^{*} \mathrm{Sub}_{\mathcal{E}}$ is locally small iff $P_{F}$ is locally small (see [Str20]).

\section{Constant objects functors are not unique}

For arbitrary base toposes $\mathcal{S}$ triposes $F, G: \mathcal{S} \rightarrow \mathcal{E}$ need not be equivalent since if $\mathcal{S}$ is $\operatorname{Sh}(X)$ and $\mathcal{E}$ is $\operatorname{Sh}(Y)$ for some sober spaces $X$ and $Y$ then there are at least as many triposes $\mathcal{S} \rightarrow \mathcal{E}$ (up to equivalence) as there are continuous maps from $Y$ to $X$. But even if $\mathcal{S}$ is Set there are in general many non-equivalent triposes over Set giving rise to the same topos as shown by the following simple counterexample

Theorem 3.1. For every natural number $n>0$ the functor $F_{n}$ : Set $\rightarrow$ Set $: I \mapsto$ $I^{n}$ is a tripos. The triposes $F_{n}^{*} \operatorname{Sub}_{\mathbf{S e t}}$ and $F_{m}^{*}$ Sub $_{\text {Set }}$ are equivalent if and only if $n=m$.

Proof. Obviously, the $F_{n}$ preserve finite limits since they are right adjoints and every $I \in$ Set appears as split subobject of $F_{n}(I)$. Thus, all $F_{n}$ are triposes but $F_{n}^{*}$ Sub $_{\text {Set }}$ and $F_{m}^{*}$ Sub $_{\text {Set }}$ are equivalent as triposes if and only if $n=m$ since the latter is equivalent to $2^{n}=2^{m}$ which in turn is equivalent to $F_{n}^{*} \operatorname{Sub} \operatorname{Set}(2) \simeq$ $F_{m}^{*} \operatorname{Sub}_{\text {Set }}(2)$.

Notice, however, that $F_{n}$ is a traditional tripos if and only if $n=1$. Thus, it may still be the case that there exist traditional triposes $F, G: \mathcal{S} \rightarrow \mathcal{E}$ which are not equivalent as triposes. Unfortunately, we have not been able so far to find examples of non-equivalent traditional triposes $\mathscr{P}_{1}$ and $\mathscr{P}_{2}$ over Set such that the ensuing toposes $\operatorname{Set}\left[\mathscr{P}_{1}\right]$ and $\operatorname{Set}\left[\mathscr{P}_{2}\right]$ are equivalent (a question which for the special case of localic toposes has been raised already in HJP80, p. 228]). However, though a bit annoying, we can't find this as a major problem since our weak notion of tripos is conceptually more adequate than the traditional one because from a logical point of view adding the comprehension axiom to first-order posetal hyperdoctrines appears much more natural than requiring that they are witnessed by Skolem functions in the base $\mathcal{S}$, i.e. requiring for all $\rho \in \mathscr{P}(I \times J)$ the existence of a function $r: J \rightarrow P(I)$ such that

$$
\forall j \in J . \forall i \in I . \rho(i, j) \leftrightarrow i \varepsilon_{I} r(j)
$$

holds in the logic of $\mathscr{P}$. At the end of Pit02 the author expresses a similar view in a slightly more cautious way.

Finally, we observe that triposes over Set may give rise to non-localic Grothendieck toposes. Let $\mathcal{E}$ be the topos of reflexive graphs, i.e. presheaves over the 3 element monoid $\Delta([1],[1])$ of monotone endomaps of the ordinal 2 . As observed by Lawvere the global elements functor $\Gamma: \mathcal{E} \rightarrow$ Set fits into a sequence of adjoints $\Pi \dashv \Delta \dashv \Gamma \dashv \nabla$ : Set $\hookrightarrow \mathcal{E}$. The rightmost functor $\nabla$ preserves all limits since it has a left adjoint. Subobjects of objects of the form $\nabla(I)$ are up to isomorphism precisely those reflexive graphs where between two nodes there is at most one edge (i.e. directed graphs as traditionally considered in combinatorics!). But since any reflexive graph can be covered by such a traditional directed graph every object of $\mathcal{E}$ appears as subquotient of some $\nabla(I)$ for which reason $\nabla$ is a tripos over Set though it is not the inverse image part of a geometric morphism.

\section{Regular triposes}

It is well known that a morphism $e: Y \rightarrow X$ in an elementary topos $\mathcal{E}$ is epic iff the pullback functor $e^{*}: \operatorname{Sub}_{\mathcal{E}}(X) \rightarrow \operatorname{Sub}_{\mathcal{E}}(Y)$ reflects maximal subobjects, i.e. 
a mono $m: P \longmapsto X$ in $\mathcal{E}$ is an iso already if $e^{*} m$ is an iso. Recall that a preorder fibered over a regular category is a prestack (w.r.t. the regular cover topology) iff for all regular epis $e$ reindexing along it (preserves and) reflects the order. Thus, for a tripos $F: \mathcal{S} \rightarrow \mathcal{E}$ the fibered preorder $F^{*} \operatorname{Sub}_{\mathcal{E}}$ is a prestack iff $F$ preserves (regular) epis.

This observation strongly suggests to require that triposes $F: \mathcal{S} \rightarrow \mathcal{E}$ also preserve epis since it vacuously holds when $\mathcal{S}$ is Set (since in Set all epis are split as ensured by the axiom of choice!) and, moreover, by Lemma 6.1 ("Pitts's Iteration Theorem") of [Pit81] triposes preserving epis are closed under composition.

Definition 4.1. A tripos $F: \mathcal{S} \rightarrow \mathcal{E}$ is called regular iff $F$ preserves epis.

Recall that a functor between regular categories is called regular iff it preserves finite limits and regular epis. Thus, regular triposes are regular functors $F: \mathcal{S} \rightarrow \mathcal{E}$ between toposes such that every $A \in \mathcal{E}$ appears as subquotient of $F I$ for some $I \in \mathcal{S}$. The usual proof (as in [Joh77]) that localic geometric morphisms are closed under composition extends straightforwardly to an argument showing that regular triposes are closed under composition

From Prop. 3.14 of Pit81 it follows that a traditional tripos $F: \mathcal{S} \rightarrow \mathcal{E}$ is regular iff it has "fibrewise quantification", i.e. there are maps $\bigvee, \wedge: \Omega_{\mathcal{S}}^{\Sigma} \rightarrow \Sigma$ such that $\exists_{F u}(F p)^{*} t$ and $\forall_{F u}(F p)^{*} t$ appear as pullbacks of $t: T \longmapsto F \Sigma$ along $F(\lambda i: I . \bigvee\{p(j) \mid u(j)=i\})$ and $F(\lambda i: I . \bigwedge\{p(j) \mid u(j)=i\})$, respectively, for all $u: J \rightarrow I$ and $p: J \rightarrow \Sigma$.

Theorem 4.2. Let $F_{1}: \mathcal{S} \rightarrow \mathcal{E}_{1}$ and $F_{2}: \mathcal{S} \rightarrow \mathcal{E}_{2}$ be triposes and $H: F_{1} \rightarrow F_{2}$, i.e. $H: \mathcal{E}_{1} \rightarrow \mathcal{E}_{2}$ with $F_{2}=H F_{1}$. Then $H$ is a tripos iff $H$ preserves finite limits and $H$ is a regular tripos iff $H$ is a regular functor.

Proof. The forward directions are trivial. For the backwards directions suppose $A \in \mathcal{E}_{2}$. Then, since $F_{2}$ is a tripos there exists a subobject $m: C \mapsto F_{2} I$ and an epi $e: C \rightarrow A$. Since $F_{2}=H F_{1}$ we have $m: C \nrightarrow H\left(F_{1} I\right)$ and $e: C \rightarrow A$. Thus, we have shown that $H$ validates the second condition required for a tripos.

The previous theorem for regular triposes $F_{1}: \mathcal{S} \rightarrow \mathcal{E}_{1}$ and $F_{2}: \mathcal{S} \rightarrow \mathcal{E}_{2}$ suggests that the right notion of morphism from $F_{1}$ to $F_{2}$ is a functor $H: F_{1} \rightarrow F_{2}$ such that $H: \mathcal{E}_{1} \rightarrow \mathcal{E}_{2}$ is regular since for this definition morphisms to a regular tripos $F: \mathcal{S} \rightarrow \mathcal{E}$ coincide with regular triposes over $\mathcal{E} \mathbb{2}^{2}$

In the subsequent Theorem 4.4 we will show that morphisms between traditional regular triposes are precisely the traditional regular triposes. But for this purpose we need the following lemma characterizing traditional regular triposes among regular triposes in terms of a condition which at first sight looks weaker than the one given in Def. 2.1

\footnotetext{
${ }^{2}$ Moreover, for a not necessarily regular tripos $F: \mathcal{S} \rightarrow \mathcal{E}$ a regular functor $H$ from $\mathcal{E}$ to a topos $\mathcal{F}$ is a regular tripos whenever $H \circ F: \mathcal{S} \rightarrow \mathcal{F}$ is a regular tripos.
} 
Lemma 4.3. Let $F: \mathcal{S} \rightarrow \mathcal{E}$ be a regular tripos and $t: T \longmapsto F \Sigma$ be weakly generic for $F^{*} \mathrm{Sub}_{\mathcal{E}}$, i.e. every mono $m: P \longmapsto F I$ fits into a diagram

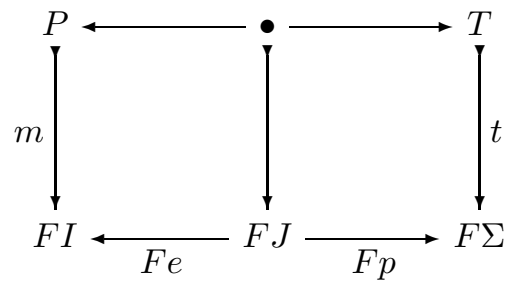

where both squares are pullbacks and $e: J \rightarrow I$ is epic, then $F$ is a traditional tripos.

Proof. Suppose $t: T \longmapsto F \Sigma$ is a weakly generic family for $F^{*}$ Sub S $_{\mathcal{E}}$. Let $E=$ $\{(u, U) \in \Sigma \times P(\Sigma) \mid u \in U\}$ and $p: E \rightarrow \Sigma$ and $q: E \rightarrow P(\Sigma)$ the respective projection maps. We will show that $\exists_{q} p^{*} t$ is a generic family for $F^{*} \operatorname{Sub}_{\mathcal{E}}$.

For this purpose suppose $m \in \operatorname{Sub}_{\mathcal{E}}(F I)$. By assumption there are $e: J \rightarrow I$ and $f: J \rightarrow \Sigma$ such that $e^{*} m \cong f^{*} t$. Since $F^{*} \operatorname{Sub}_{\mathcal{E}}$ is a prestack w.r.t. the regular cover topology we have $m \cong \exists_{e} e^{*} m \cong \exists_{e} f^{*} t$. Let $g: I \rightarrow P(\Sigma)$ with $g(i)=\{f(j) \mid e(j)=i\}$. Obviously, the map $\langle f, g e\rangle$ factors through $\langle p, q\rangle$ since $f(j) \in g(e(j))$. Consider the following diagram

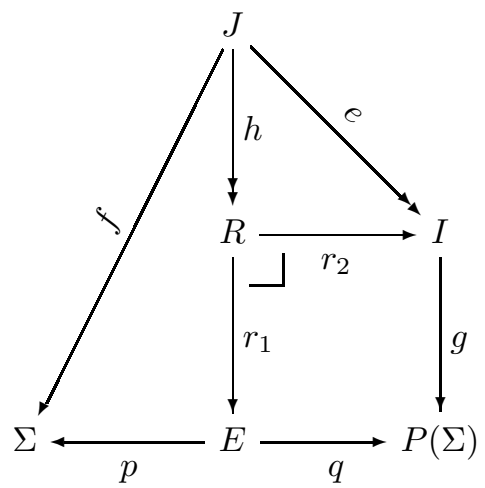

where $R=\{(u, U, i) \in \Sigma \times P(\Sigma) \times I \mid u \in U=g(i)\}$ with $r_{1}$ and $r_{2}$ the respective projections and $h(j)=(f(j), g(e(j)), e(j))$. Notice that $h$ is onto since $g(i)=$ $\{f(j) \mid e(j)=i\}$. We have

$$
\begin{aligned}
g^{*} \exists_{q} p^{*} t & \cong \exists_{r_{2}} r_{1}^{*} p^{*} t & & \text { by Beck-Chevalley condition } \\
& \cong \exists_{r_{2}} \exists_{h} h^{*} r_{1}^{*} p^{*} t & & \text { since } h \text { is epic } \\
& \cong \exists_{e} f^{*} t & & \text { since } e=r_{2} h \text { and } f=p r_{1} h \\
& \cong m & &
\end{aligned}
$$

as desired.

Theorem 4.4. Let $F_{1}: \mathcal{S} \rightarrow \mathcal{E}_{1}$ be a traditional regular tripos and $H: \mathcal{E}_{1} \rightarrow \mathcal{E}_{2}$ a regular functor between toposes. Then $H$ is a traditional regular tripos if and only if $F_{2}=H F_{1}$ is a traditional regular tripos.

Proof. The forward direction is Pitts's Iteration Theorem.

For the backward direction suppose that $H: \mathcal{E}_{1} \rightarrow \mathcal{E}_{2}$ is a regular functor such that $F_{2}=H F_{1}$ is a traditional regular tripos. By Theorem 4.2 it is immediate that $H$ is a regular tripos, too. Since $F_{2}$ has been assumed to be a traditional tripos 
there is a $t: T \longmapsto F_{2} \Sigma$ generic for $F_{2}^{*} \operatorname{Sub}_{\mathcal{E}_{2}}$. For showing that $H$ is a traditional tripos it suffices by Lemma 4.3 to show that $t: T \longmapsto H F_{1} \Sigma$ is weakly generic for $H^{*} \operatorname{Sub}_{\mathcal{E}_{2}}$.

Suppose $m: P \longmapsto H A$ for some $A \in \mathcal{E}_{1}$. Since $F_{1}$ is a traditional tripos there exist $n: Q \longmapsto F_{1} I$ and $e: Q \rightarrow A$ for some $I \in \mathcal{S}$. Since $F_{2}=H F_{1}$ is a traditional tripos there exists $p: I \rightarrow \Sigma$ such that $H n \circ(H e)^{*} m$ arises as pullback of $t$ along $H F_{1} p$ for some $p: I \rightarrow \Sigma$. Thus we have

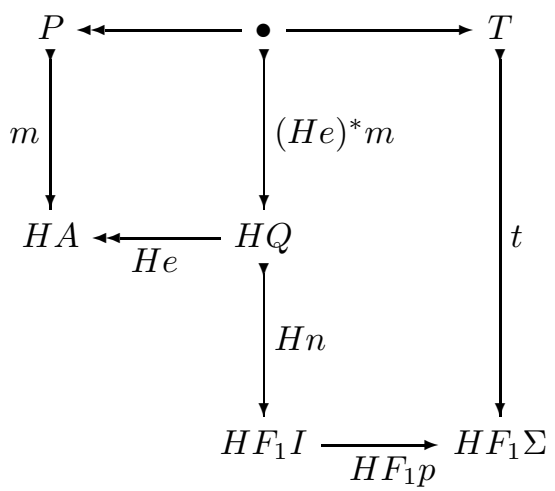

from which it follows that $(H e)^{*} m$ arises as pullback of $t$ along $H\left(F_{1} p \circ n\right)=$ $H F_{1} p \circ H n$. Thus, we have

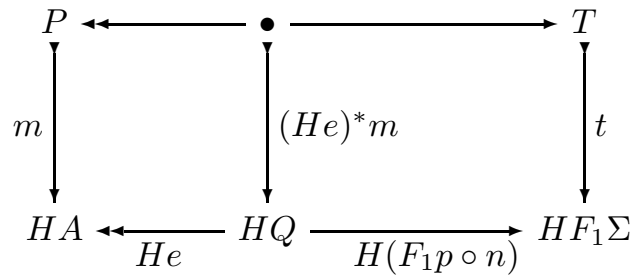

where both squares are pullbacks as required.

We conclude this section with some observations on the

4.1. Preservation of assemblies by tripos morphisms. Following vO08 for a tripos $F: \mathcal{S} \rightarrow \mathcal{E}$ one may define assemblies as those objects of $\mathcal{E}$ which appear as subobjects of some $F I$. If $G: \mathcal{S} \rightarrow \mathcal{F}$ is a tripos and $H: F \rightarrow G$ such that $H: \mathcal{E} \rightarrow \mathcal{F}$ preserves finite limits then $H$ preserves assemblies, i.e. sends assemblies w.r.t. $F$ to assemblies w.r.t. $G$, since $H m: H P \longmapsto H F I=G I$ whenever $m: P \longmapsto$ $F I$. It follows from the definition of tripos that every object $A$ of $\mathcal{E}$ appears as subquotient of some $F I$, i.e. we have $A \stackrel{e}{\longleftarrow} C \stackrel{m}{\longrightarrow}$ FI. If $H: F \rightarrow G$ is a regular functor between triposes then $H A \stackrel{H e}{\longleftarrow} H C \stackrel{H m}{\longrightarrow} H F I=G I$, i.e. $H$ preserves coverings of objects by assemblies in a very strong sense.

\section{Relation to Miquel's implicative algebras}

In Miq20b A. Miquel has shown that traditional triposes over Set correspond to so called implicative algebras Miq20a.

Definition 5.1. An implicative structure is a complete lattice $\mathcal{A}$ together with an operation $\rightarrow: \mathcal{A}^{\mathrm{op}} \times \mathcal{A} \rightarrow \mathcal{A}$ such that $x \rightarrow \bigwedge Y=\bigwedge_{y \in Y}(x \rightarrow y)$ for all $x \in \mathcal{A}$ and 
$Y \subseteq \mathcal{A}$. Then $K_{\mathcal{A}}=\bigwedge_{x, y \in \mathcal{A}} x \rightarrow y \rightarrow x$ and $S_{\mathcal{A}}=\bigwedge_{x, y, z \in \mathcal{A}}(x \rightarrow y \rightarrow z) \rightarrow(x \rightarrow y) \rightarrow x \rightarrow z$ are elements of $\mathcal{A}$.

$A$ separator in an implicative structure $(\mathcal{A}, \rightarrow)$ is an upward closed subset $\mathcal{S}$ of $\mathcal{A}$ such that $K_{\mathcal{A}}, S_{\mathcal{A}} \in \mathcal{S}$ and $\mathcal{S}$ is closed under modus ponens, i.e. $b \in \mathcal{S}$ whenever $a \in \mathcal{S}$ and $a \rightarrow b \in \mathcal{S}$.

An implicative algebra is a triple $(\mathcal{A}, \rightarrow, \mathcal{S})$ such that $(\mathcal{A}, \rightarrow)$ is an implicative structure and $\mathcal{S}$ is a separator in $(\mathcal{A}, \rightarrow)$.

With every implicative algebra $\mathcal{A}$ one associates a Set-based tripos $\mathscr{P} \mathcal{A}$ where $\mathscr{P} \mathcal{A}^{\mathcal{A}}(I)$ is the preorder $\vdash_{I}$ on $\mathcal{A}^{I}$ defined as

$$
\varphi \vdash_{I} \psi \quad \text { iff } \bigwedge_{i \in I}\left(\varphi_{i} \rightarrow \psi_{i}\right) \in \mathcal{S}
$$

and reindexing is given by precomposition.

In Miq20b A. Miquel has shown that every traditional regular tripos over Set is equivalent to $\mathscr{P} \mathcal{A}$ for some implicative algebra $\mathcal{A}$.

For $i=1,2$ let $F_{i}:$ Set $\rightarrow \mathcal{E}_{i}$ be the constant objects functor for the regular tripos induced by an implicative algebra $\mathcal{A}_{i}$ in Set, i.e. $\mathcal{E}_{i}=\operatorname{Set}\left[\mathscr{P} \mathcal{A}_{i}\right]$. Due to the remark in Subsection 4.1 regular functors $G: \mathcal{E}_{1} \rightarrow \mathcal{E}_{2}$ with $F_{2}=G F_{1}$ correspond to cartesian functors $g: F_{1}^{*} \operatorname{Sub}_{\mathcal{E}_{1}} \rightarrow F_{2}^{*} \operatorname{Sub}_{\mathcal{E}_{2}}$ preserving regular logic, i.e. finite limits and existential quantification. Obviously, such $g$ are uniquely determined by $h=g_{\mathcal{A}_{1}}\left(\operatorname{id}_{\mathcal{A}_{1}}\right): \mathcal{A}_{1} \rightarrow \mathcal{A}_{2}$ since $g_{I}\left(\varphi: I \rightarrow \mathcal{A}_{1}\right)=h \circ \varphi$. This suggests to define a morphism of implicative algebras from $\mathcal{A}_{1}$ to $\mathcal{A}_{2}$ as a function $h: \mathcal{A}_{1} \rightarrow \mathcal{A}_{2}$ such that the cartesian functor $g: F_{1}^{*} \operatorname{Sub}_{\mathcal{E}_{1}} \rightarrow F_{2}^{*} \operatorname{Sub}_{\mathcal{E}_{2}}$ given by $g_{I}\left(\varphi: I \rightarrow \mathcal{A}_{1}\right)=h \circ \varphi$ preserves regular logic, i.e. finite limits and existential quantification.

Unfortunately, Miquel's result from Miq20b does not extend to arbitrary base toposes. The reason is that for a traditional regular tripos $F: \mathcal{S} \rightarrow \mathcal{E}$ there need not exist a subobject $S$ of $\Sigma$ such that

(1) its characteristic map $\chi_{S}: \Sigma \rightarrow \Omega_{\mathcal{S}}$ induces by postcomposition a cartesian functor $\gamma_{S}: F^{*} \operatorname{Sub}_{\mathcal{E}} \rightarrow$ Sub $_{\mathcal{S}}$ preserving finite meets in each fiber and

(2) $u: 1 \rightarrow \Sigma$ factors through $S$ iff $(F u)^{*} t$ is isomorphic to $\operatorname{id}_{F 1}$.

Notice that the first condition means that $u^{*} S \leq v^{*} S$ whenever $F(u)^{*} t \leq F(v)^{*} t$ and that $t \in S$ and $\forall u, v: \Sigma . u \wedge v \in S \leftrightarrow(u \in S \wedge v \in S)$ hold in the internal logic of $\mathcal{S}$. For base toposes $\mathcal{S}$ which are not well-pointed such $S$ need neither exist nor be unique (for the latter see Example 4.12.12 of Fre14 for a counterexample 3 ).

The related stronger condition that $u: I \rightarrow \Sigma$ factors through $S$ iff $F(u)^{*} t$ is isomorphic to $\mathrm{id}_{F I}$ is known as "definability of truth", i.e. that the full subfibration of $F^{*} \mathrm{Sub}_{\mathcal{E}}$ on true predicates is definable in the sense of Bénabou (see Section 12 of $[\operatorname{Str20}$ ). This stronger condition, however, amounts to the requirement that the fibration $F^{*} \mathrm{Sub}_{\mathcal{E}}$ is locally small, i.e. equivalent to the externalization of a complete Heyting algebra internal to $\mathcal{S}$, which in turn is equivalent to the requirement that $F$ is the inverse image part of a localic geometric morphism.

\footnotetext{
${ }^{3}$ Take for $\mathcal{S}$ the Sierpiński topos $\operatorname{Set}^{\mathcal{T}^{\text {op }}}$ and for $F$ the functor $\operatorname{Id}_{\mathcal{S}}$. Then there are two possible choices for $S$, namely $T: 1 \rightarrow \Omega_{\mathcal{S}}$ and the subobject $S$ of $\Omega_{\mathcal{S}}$ with $S_{0}=\Omega_{0}$ and $S_{1}=\{\top\}$. In the first case the corresponding $\gamma_{S}$ is $\operatorname{id}_{\Omega_{\mathcal{S}}}$ and in the second case it sends a subobject $P$ of $A$ in $\mathcal{S}$ to the subobject $\gamma_{S}(P)$ of $A$ with $\gamma_{S}(P)_{0}=A_{0}$ and $\gamma_{S}(P)_{1}=P_{1}$.
} 


\section{Summary and Conclusion}

We have shown in which sense (generalized) triposes in the sense of Pit81 may be understood as a generalizations of localic geometric morphisms. The traditional triposes of HJP80 can be characterized as those triposes $F: \mathcal{S} \rightarrow \mathcal{E}$ for which the fibered preorder $F^{*} \operatorname{Sub}_{\mathcal{E}}$ admits a generic family $t: T \longmapsto F \Sigma$.

We have defined regular triposes as triposes $F: \mathcal{S} \rightarrow \mathcal{E}$ where $F$ preserves epis, i.e. $F^{*} \mathrm{Sub}_{\mathcal{E}}$ is a prestack. As opposed to ordinary triposes regular triposes are known to be closed under composition, i.e. are closed under iteration. A further advantage of regular triposes is that for a regular tripos $F: \mathcal{S} \rightarrow \mathcal{E}$ regular triposes over $\mathcal{E}$ correspond to morphisms of regular triposes from $F$ to some regular tripos $G: \mathcal{S} \rightarrow \mathcal{F}$, i.e. $H: F \rightarrow G$ such that $H: \mathcal{E} \rightarrow \mathcal{F}$ is a regular functor. Somewhat surprisingly, an analogous result holds for traditional regular triposes as well.

Finally, we have recalled a theorem due to A. Miquel characterizing traditional regular triposes in terms of implicative algebras generalizing the notion of complete Heyting algebra and identified a notion of morphism between implicative algebras corresponding to regular morphisms of triposes over Set.

We think that the more general notion of tripos as introduced in Pit02 is more natural since it corresponds to the class of first-order posetal hyperdoctrines which give rise to toposes by "adding subquotients". Moreover, the comprehension axiom characterizing them is more natural than the Skolemized form postulated as an axiom in the definition of traditional triposes.

But restricting to regular triposes seems to be a good idea since the condition is most natural from the point of view of fibered categories and, moreover, allows one to identify regular tripos morphisms to $F: \mathcal{S} \rightarrow \mathcal{E}$ with regular triposes over $\mathcal{E}$ as shown in Theorem 4.2 .

We have shown that triposes $F, G$ : Set $\rightarrow \mathcal{E}$ need not be equivalent. But we do not know whether such $F$ and $G$ are necessarily equivalent under the stronger assumption that both $F$ and $G$ are traditional triposes. There is no conceptual reason why this should hold in general but, alas, we have not been able to find a counterexample so far.

Acknowledgements. We thank A. Miquel for making an early version of Miq20b available to us. The second named author thanks S. Maschio for discussions which have triggered the identification of the right notion of morphism between triposes. We further acknowledge the use of Paul Taylor's diagram macros used for writing this paper. The first named author gratefully acknowledges support by the Air Force Office of Scientific Research through grant FA9550-20-1-0305 and MURI grant FA9550-15-1-0053. 


\section{REFERENCES}

[Bén74] J. Bénabou, Logique catégorique, Lecture notes of a course at Univ. Montreal (1974).

[Bén80] Des catégories fibrées, Handwritten Lecture Notes by J.-R. Roisin of a course at Univ. Louvain-la-Neuve. (1980).

[Fre14] J. Frey, A fibrational study of realizability toposes, Ph.D. thesis, Paris 7 University, 2014, arXiv: 1403.3672 .

[HJP80] J. Hyland, P. Johnstone, and A. Pitts, Tripos theory, Math. Proc. Cambridge Philos. Soc. 88 (1980), no. 2, 205-232.

[Jib88] M. Jibladze, Geometric morphisms and indexed toposes, Proc. Conf. Cat. Top. Prague, 1988, pp. 10-18.

[Joh77] P. Johnstone, Topos theory, Academic Press, 1977.

[Miq20a] A. Miquel, Implicative algebras: a new foundation for realizability and forcing, Math. Struct. in Computer Science 30 (2020), no. 5, 458-510.

[Miq20b] _, Implicative algebras II: Completeness w.r.t. Set-based triposes, arXiv:2011.09085 (2020).

[Pit81] A. Pitts, The theory of triposes, Ph.D. thesis, University of Cambridge, 1981.

[Pit02] Tripos theory in retrospect, Math. Struct. in Computer Science 12 (2002), no. 3, 265-279.

[Str20] T. Streicher, Fibred categories à la Jean Bénabou, arXiv:1801.02927 (2020).

[vO08] J. van Oosten, Realizability: an introduction to its categorical side, Elsevier, 2008. 